APJ ACCEPTED [26 JULY 2012]

Preprint typeset using LTEX style emulateapj v. 5/2/11

\title{
MEASUREMENT OF THE MASS AND STELLAR POPULATION DISTRIBUTION IN M82 WITH THE LBT
}

\author{
Johnny P. GReco, PAUl Martini, AND TOdD A. ThOMPSON \\ Department of Astronomy, The Ohio State University, Columbus, OH 43210, USA; greco.40@buckeyemail.osu.edu
} APJ ACCEPTED [26 JULY 2012]

\begin{abstract}
We present a $K$-band spectroscopic study of the stellar and gas kinematics, mass distribution, and stellar populations of the archetypical starburst galaxy M82. Our results are based on a single spectrum at a position angle of $67.5^{\circ}$ through the $K$-band nucleus. We used the ${ }^{12} \mathrm{CO}$ stellar absorption band head at $2.29 \mu \mathrm{m}\left(\mathrm{CO}_{2.29}\right)$ to measure the rotation curve out to nearly $4 \mathrm{kpc}$ radius on both the eastern and western sides of the galaxy. Our data show that the rotation curve is flat from $1-4 \mathrm{kpc}$. This stands in sharp contrast to some previous studies, which have interpreted $\mathrm{HI}$ and $\mathrm{CO}$ emission-line position-velocity diagrams as evidence for a declining rotation curve. The kinematics of the $\mathrm{Br} \gamma, \mathrm{H}_{2}$, and $\mathrm{He} \mathrm{I}$ emission lines are consistent with, although characterized by slightly higher velocities than, the stellar kinematics. We derived M82's mass distribution from our stellar kinematic measurements and estimate its total dynamical mass is $\sim 10^{10} M_{\odot}$. We measured the equivalent width of $\mathrm{CO}_{2.29}\left(\mathrm{~W}_{2.29}\right)$ as a function of distance from the center of the galaxy to investigate the spatial extent of the red supergiant (RSG) population. The variation in $\mathrm{W}_{2.29}$ with radius clearly shows that RSGs dominate the light inside $500 \mathrm{pc}$ radius. M82's superwind is likely launched from this region, where we estimate the enclosed mass is $\lesssim 2 \times 10^{9} M_{\odot}$.

Subject headings: galaxies: individual (M82) - galaxies: starburst - galaxies: kinematics and dynamics infrared: galaxies
\end{abstract}

\section{INTRODUCTION}

Observations suggest that star formation in galaxies is regulated by feedback processes. In rapidly star-forming galaxies, feedback operates on large scales, driving galaxy-wide superwinds, which can eject the raw materials for future stellar generations if the interstellar material is launched with enough momentum to escape the host galaxy's gravitational potential. Otherwise, the ejected material may be re-accreted in a so-called "fountain flow". These issues are crucial for understanding the formation of stars in rapidly star-forming galaxies across cosmic time.

The exceptionally high star formation rates present in starburst galaxies make them optimal targets to probe feedback processes. The focus of this paper is the archetype of this class of objects, M82. This galaxy's proximity (3.63 Mpc; $1^{\prime \prime}=17.6 p c$; Freedman et al. 1994; Sakai \& Madore 1999; Gerke et al. 2011) and nearly edge-on geometry $\left(i=80^{\circ}\right.$; Lynds \& Sandage 1963; McKeith et al. 1995) make it a superb laboratory for studying the physics of galactic winds and star formation. At infrared wavelengths, its luminosity is $6 \times 10^{10} L_{\odot}($ Sanders et al. 2003), which makes it one of the brightest and most observed infrared objects in the sky. Most of its infrared luminosity originates in the starburst core, which is severely obscured by dust at visible and ultraviolet wavelengths (Rieke et al. 1980; Förster Schreiber et al. 2001).

The origin of M82's starburst is thought to be a tidal interaction with its more massive companion, M81, $10^{8}$ years ago (Gottesman \& Weliachew 1977; Lo et al. 1987; Telesco et al. 1991; Achtermann \& Lacy 1995). The pronounced HI bridge that connects the galaxies (Cottrell 1977; Yun et al. 1993) is strong evidence for such an interaction, and Yun et al. (1994) found that the "cloud of H I" that engulfs the M81 group is dominated by filamentary tidal structures, which suggests a violent past of tidal interaction. In addition to the dynamical effects associated with tidal forces from M81, the global dy- namics and evolution of the starburst are further perturbed by the presence of a stellar bar (Telesco et al. 1991), which may have formed as a result of the interaction.

As an interacting, barred galaxy, M82's observed dynamical properties are distinct from those seen in typical $\mathrm{Sb}$ and $\mathrm{Sc}$ galaxies. Molecular gas measurements by Young \& Scoville (1984) show that the CO velocity field contains warps and peculiarities that are consistent with a significant non-circular velocity component, perhaps due to radial infall or a polar ring tilted with respect to the major axis. In addition, measurements of CO $(2,1)$ by Sofue et al. (1992) suggest that the rotation of the gas disk is nearly Keplerian at radii $>1 \mathrm{kpc}$. Sofue (1998) reproduced this Keplerian motion with a model in which the outer disk and halo were stripped during the encounter with M81. Although flat rotation curves are observed in the vast majority of galaxies, Rubin \& Ford (1983) found falling rotation curves in several tidally interacting galaxies, which suggests that some dark matter is stripped from galaxies during interactions.

The $\mathrm{H}$ I velocity field along the major axis is similar to the $\mathrm{CO}$ velocity field; it also has peculiarities such as a sudden drop at $\sim 1 \mathrm{kpc}$, which some authors have interpreted as a falling rotation curve (Yun et al. 1993). It is important to note, however, that the H I gas is highly disrupted and may not provide a reliable rotation curve for the outer disk. In fact, Yun et al. (1993) state that rotation dominates the H I kinematic signature inside the central $1 \mathrm{kpc}$, but the outer region is characterized by a significant, likely tidally induced, nonrotational component.

The gas and stars in M82 have been shown to be composed of two distinct kinematic structures. Westmoquette et al. (2009) found that the axis of gas rotation is offset from the stellar rotation axis and the photometric major axis by $\sim 12^{\circ}$. This result implies that, in long-slit spectroscopic studies, the shape of the rotation curve is particularly sensitive to the position and alignment of the slit. In addition, 
Konstantopoulos et al. (2009) studied M82's star cluster population out to $\sim 2.6 \mathrm{kpc}$ radius and found that the rotation curve as traced by the clusters is flat at large radii, which further distinguishes the stellar kinematic tracers from the previous measurements of the gas kinematics.

Förster Schreiber et al. (2003) proposed that M82's starburst activity occurred in two successive episodes that each lasted a few million years and peaked about $10^{7}$ and $5 \times 10^{6}$ years ago. In this model, the encounter with M81 triggered the first burst in the central $500 \mathrm{pc}$ by inducing strong largescale torques, loss of angular momentum, and an infall of molecular clouds toward the nuclear region. The second burst occurred in a circumnuclear ring and along the stellar bar and is attributed to bar-induced dynamical resonances. The Förster Schreiber et al. (2003) starburst model can explain the apparent abundance of red supergiants (RSGs) that dominate the light within $500 \mathrm{pc}$ radius.

In order to understand the dynamics of the ejected gas and constrain the physics of feedback processes in M82, it is essential to accurately measure the total dynamical mass on both the small scales of star-forming regions and the large scales of the entire galaxy. Rotation curves are most commonly employed to determine the distribution of mass in galaxies (Sofue \& Rubin 2001), and many studies have employed gas and stellar kinematics at a range of wavelengths to measure radial velocities in M82 (e.g., Saito et al. 1984; Goetz et al. 1990; McKeith et al. 1993; Achtermann \& Lacy 1995; Sofue et al. 1992). Greve (2011) includes a compilation of these results. Given that M82 has recently undergone tidal interactions and harbors an extraordinary superwind, stellar kinematics should be a more reliable tracer of the mass distribution than the gas. Nearly all previous stellar kinematic measurements used visible-wavelength tracers, which may be compromised by the substantial dust attenuation present in the galaxy. The near-infrared $K-$ band is a better tracer of the stellar kinematics, as attenuation $A_{\lambda}$ in the $K$-band is approximately a factor of 10 less than in the $V$-band. In this study, we use $K$-band spectroscopy to study M82's gas and stellar kinematics, mass distribution, and stellar populations.

\section{OBSERVATIONS AND DATA REDUCTION}

We obtained $K$-band spectra of M82 at the Mount Graham International Observatory with the LUCI-1 spectrograph and the Large Binocular Telescope (LBT) on 2011 February 10. The observations were made through a $K$-band filter (2.05$2.37 \mu \mathrm{m}$ ) with the N1.8 camera, which has a plate scale of $0^{\prime \prime} .25$ pixel $^{-1}$ and a field of view of $4^{\prime} \times 4^{\prime}$. A grating with 210 lines $\mathrm{mm}^{-1}$ was used with a slit width of $1^{\prime \prime}$. This combination yields a spectral resolution of $\lambda / \Delta \lambda \approx 3000$ or 100 $\mathrm{km} \mathrm{s}^{-1}$. With sufficient signal-to-noise, the centroid of the cross correlation function for relative velocity measurements can be measured with much higher resolution. Two slit positions, which we designate as M82E and M82W, were used to measure the rotation curve out to $4^{\prime}(\sim 4 \mathrm{kpc})$ from the center of the galaxy. We centered each end of the slit on the nuclear region at a position angle of $67.5^{\circ}$ (Figure 1). The observations were made with 300 second exposures in an object-sky sequence for a total on-source integration time of 1 hour per slit position. A telluric standard close in air mass to M82 was observed before and after both the M82E and M82W observations.

We carried out standard data reduction with the
IRAF 1 software package. The two-dimensional data were transformed onto an orthogonal wavelength/slit-position grid based on a wavelength solution from the sky's bright $\mathrm{OH}$ emission lines. This transformation linearized the dispersion to $\sim 1.64 \AA$ pixel $^{-1}$. We used the xtell cor (Vacca et al. 2003) package in IDL to correct for absorption from Earth's atmosphere. The telluric standard spectra had slightly higher spectral resolution than was obtained for M82. This is likely because the light from the stars did not fill the $1^{\prime \prime}$ wide slit. This complication was remedied by convolving the telluric spectra with Gaussians to match their resolutions to M82.

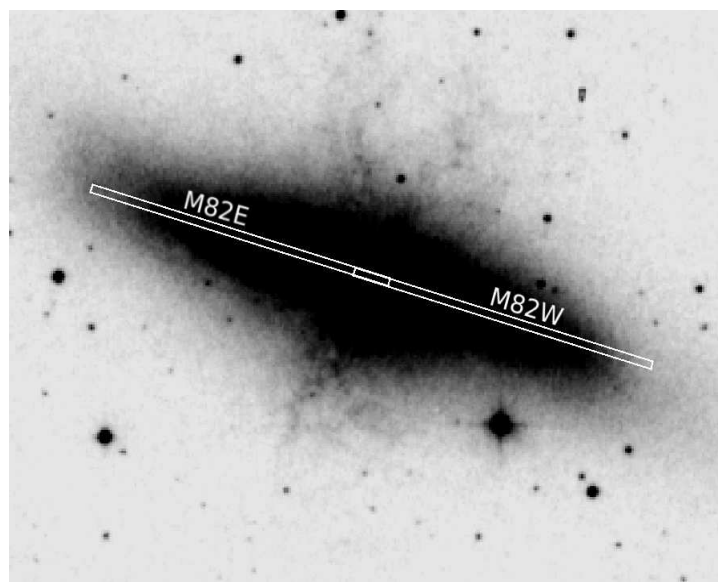

FIG. 1.- Digital Sky Survey image of M82 with overlays of our two slit positions. Each slit is $4^{\prime}$ long and they overlap on the center of the galaxy. The widths of the slits have been increased by a factor of six for ease of visibility in this figure. North is up and East is to the left.

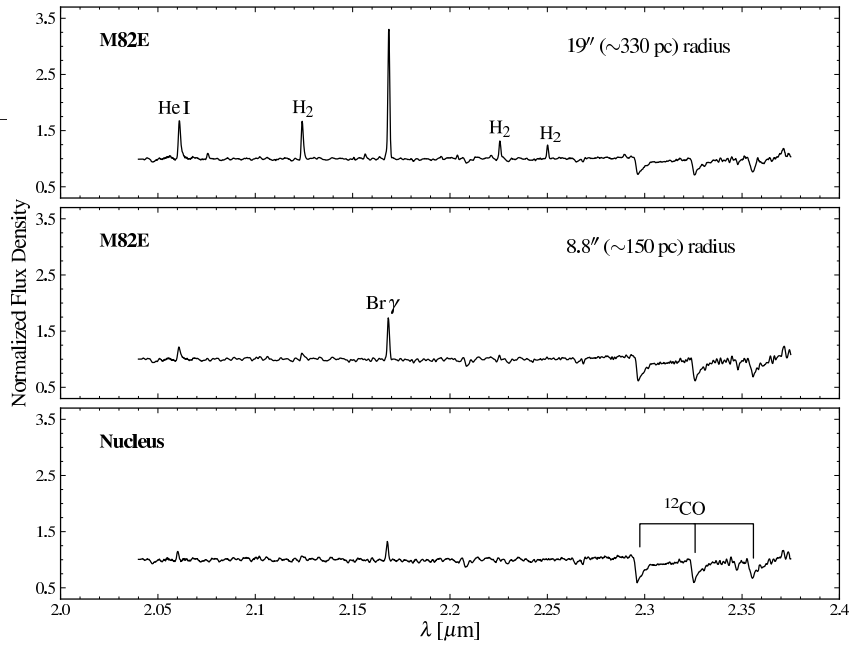

FIG. 2.- $K$-band spectra from M82's nucleus, $8.8^{\prime \prime}$ east from center, and $19^{\prime \prime}$ east from center. $\mathrm{CO}_{2.29}$, which is the dominant absorption feature, originates in the atmospheres of cool, evolved stars. The He I and $\mathrm{Br} \gamma$ lines trace star formation, and the $\mathrm{H}_{2}$ is evidence of shocked gas. The strength of the emission lines peaks near $19^{\prime \prime}$.

\section{ROTATION CURVE}

To measure the rotation curve, we symmetrically extracted 30 apertures each from the M82E and M82W slit positions.

1 IRAF is distributed by the National Optical Astronomy Observatory, which is operated by the Association of Universities for Research in Astronomy, Inc., under cooperative agreement with the National Science Foundation. 


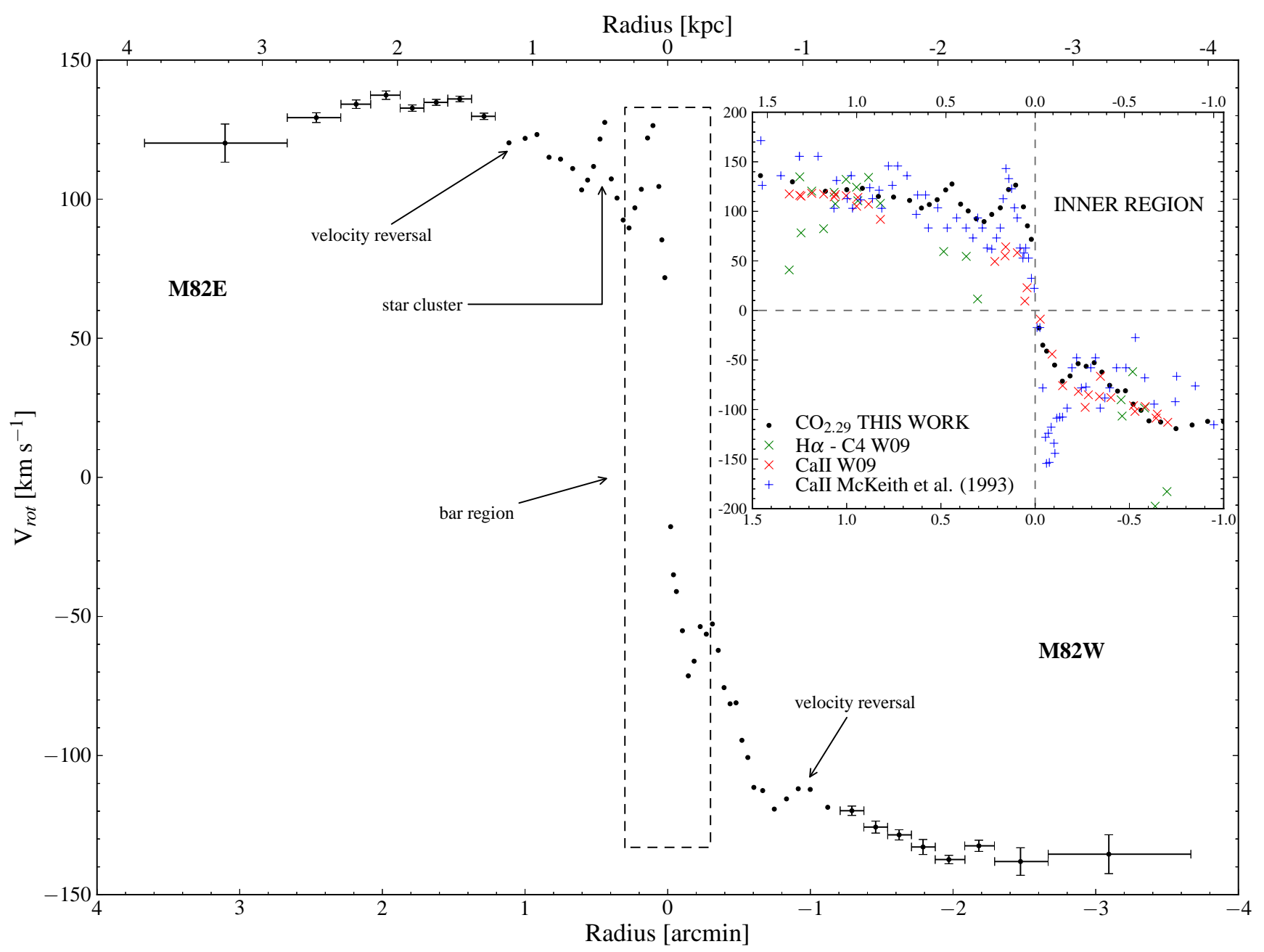

FIG. 3. - Stellar rotation curve based on $\mathrm{CO}_{2.29}$. The horizontal error bars indicate the aperture size of the measurement, and the vertical error bars represent the rms scatter between the six cross correlation templates. All data points without error bars have uncertainties less than $2 \mathrm{~km} \mathrm{~s}^{-1}$ and aperture sizes less than $5^{\prime \prime}$. We assume an inclination of $80^{\circ}$ for all velocity measurements. The symmetric, sharp features on either side of the nucleus are due to the non-circular motions of the stellar bar. At $\sim 1 \mathrm{kpc}$ on either side of the galaxy, there is an apparent "velocity reversal", which may be due to symmetric spiral arms. The inset compares our results of the inner region with stellar velocity measurements by Westmoquette et al. (W09;2009) and McKeith et al. (1993). The C4 label with the W09 data refers to the component of an integral field unit (IFU) observation.

We progressively increased the aperture widths at larger radii to achieve a sufficient signal-to-noise ratio in the stellar continuum. Figure 2 shows illustrative $K$-band spectra from M82's nucleus and eastern side. We measured the rotation curve out to nearly $4 \mathrm{kpc}$ radius with the ${ }^{12} \mathrm{CO}$ stellar absorption band head at $2.29 \mu \mathrm{m}\left(\mathrm{CO}_{2.29}\right)$ and to $\sim 1 \mathrm{kpc}$ radius with gas emission ( $\mathrm{He} \mathrm{I}, \mathrm{H}_{2}$, and $\mathrm{Br} \gamma$ ) from the interstellar medium. We have assumed an inclination of $80^{\circ}$ and corrected each velocity accordingly. It is important to note that the choice of dynamical center may introduce systematic uncertainties, as the photometric center does not necessarily coincide with the dynamical center. As the gas and stars trace distinct kinematic structures, we discuss each in turn.

\subsection{Stellar Absorption}

$\mathrm{CO}_{2.29}$ originates in the atmospheres of cool, evolved stars and provides a strong absorption feature for stellar velocity measurements (e.g., Gaffney et al. 1995). Previous studies of M82 have used $\mathrm{H} \alpha$ and $\mathrm{NaD}$ to measure the rotation curve with stellar kinematics (e.g. Mavall 1960; Goetz et al. 1990), yet these measurements are likely compromised by the $A_{V} \sim$ $5 \rightarrow 25$ attenuation toward the central region (Rieke et al.
1980; Lester et al. 1990; Puxley 1991). At somewhat longer wavelengths, McKeith et al. (1993) measured the stellar kinematics with Ca II stellar absorption lines $(8662,8542$, and $8498 \AA$ ). If the near-infrared extinction wavelength dependence is given by the relation $A_{\lambda} \propto \lambda^{-1.75}$ (Draine 1989), attenuation for $\mathrm{CO}_{2.29}$ is approximately a factor of five less than $\mathrm{Ca}$ II. With the copious amount of gas and dust present, an improvement at this level may reveal kinematic features that are otherwise obscured.

To measure the stellar rotation curve, we selected six apertures with high signal-to-noise as cross-correlation templates for relative velocity measurements. We used the rvsao package in IRAF to cross-correlate all 60 apertures against each of the six templates. We restricted the wavelength range from 2.28 to $2.365 \mu \mathrm{m}$ to focus the analysis on $\mathrm{CO}_{2.29}$. This resulted in six independent rotation curves. We assumed the galaxy is symmetric on large scales to determine zero velocity. After correcting for M82's nearly edge-on inclination, we took the mean velocity at each point to be the value of the rotation curve at that point, and the uncertainty was calculated as the rms scatter between the six curves. Figure 3 shows our stel- 
TABLE 1

STELLAR ROTATION CURVE DATA

\begin{tabular}{|c|c|c|c|c|c|c|c|}
\hline \multicolumn{4}{|c|}{ M82E } & \multicolumn{4}{|c|}{ M82W } \\
\hline $\begin{array}{l}\text { Position } \\
\text { (arcmin) }\end{array}$ & $\begin{array}{l}\text { Aperture } \\
\text { (arcmin) }\end{array}$ & $\begin{array}{l}\text { Velocity } \\
\left(\mathrm{km} \mathrm{s}^{-1}\right)\end{array}$ & $\begin{array}{l}\text { Uncertainty } \\
\left(\mathrm{km} \mathrm{s}^{-1}\right)\end{array}$ & $\begin{array}{l}\text { Position } \\
\text { (arcmin) }\end{array}$ & $\begin{array}{c}\text { Aperture } \\
-1 \times(\operatorname{arcmin})\end{array}$ & $\begin{array}{c}\text { Velocity } \\
\left(\mathrm{km} \mathrm{s}^{-1}\right)\end{array}$ & $\begin{array}{c}\text { Uncertainty } \\
\left(\mathrm{km} \mathrm{s}^{-1}\right)\end{array}$ \\
\hline 0.0203 & $0.0125-0.0292$ & 71.8 & 1.8 & -0.0205 & $0.0125-0.0292$ & -17.7 & 1.3 \\
\hline 0.0411 & $0.0333-0.0500^{\dagger}$ & 85.4 & 1.9 & -0.0403 & $0.0333-0.0500^{\dagger}$ & -35.0 & 1.2 \\
\hline 0.0622 & $0.0417-0.0833$ & 104.6 & 1.7 & -0.0609 & $0.0417-0.0833$ & -41.0 & 1.2 \\
\hline 0.1034 & $0.0833-0.1250$ & 126.5 & 1.2 & -0.1031 & $0.0833-0.1250$ & -55.1 & 1.0 \\
\hline 0.1412 & $0.1250-0.1667$ & 122.0 & 1.2 & -0.1443 & $0.1250-0.1667$ & -71.4 & 0.8 \\
\hline 0.1848 & $0.1667-0.2083$ & 103.6 & 1.7 & -0.1851 & $0.1667-0.2083$ & -66.1 & 2.0 \\
\hline 0.2303 & $0.2083-0.2500$ & 96.9 & 1.4 & -0.2278 & $0.2083-0.2500$ & -53.6 & 2.7 \\
\hline 0.2711 & $0.2500-0.2917$ & 89.7 & 1.3 & -0.2706 & $0.2500-0.2917$ & -56.4 & 2.2 \\
\hline 0.3121 & $0.2917-0.3333$ & 92.5 & 1.0 & -0.3133 & $0.2917-0.3333$ & -52.7 & 1.7 \\
\hline 0.3553 & $0.3333-0.3750$ & 100.4 & 1.0 & -0.3542 & $0.3333-0.3750$ & -62.2 & 1.8 \\
\hline 0.3955 & $0.3750-0.4167$ & 107.3 & 1.3 & -0.3950 & $0.3750-0.4167$ & -75.6 & 1.7 \\
\hline 0.4413 & $0.4166-0.4583$ & 127.6 & 1.2 & -0.4372 & $0.4166-0.4583$ & -81.4 & 1.6 \\
\hline 0.4747 & $0.4583-0.5000$ & 121.6 & 1.0 & -0.4789 & $0.4583-0.5000$ & -81.0 & 1.7 \\
\hline 0.5198 & $0.5000-0.5417$ & 111.8 & 1.2 & -0.5200 & $0.5000-0.5417$ & -94.5 & 1.4 \\
\hline 0.5614 & $0.5416-0.5833$ & 106.9 & 1.3 & -0.5615 & $0.5416-0.5833$ & -100.7 & 1.4 \\
\hline 0.6032 & $0.5833-0.6250^{\dagger}$ & 103.3 & 1.8 & -0.6037 & $0.5833-0.6250^{\dagger}$ & -111.4 & 1.9 \\
\hline 0.6662 & $0.6250-0.7083$ & 111.0 & 1.5 & -0.6657 & $0.6250-0.7083$ & -112.6 & 1.7 \\
\hline 0.7512 & $0.7083-0.7917$ & 114.4 & 1.5 & -0.7471 & $0.7083-0.7917$ & -119.3 & 1.4 \\
\hline 0.8318 & $0.7916-0.8750$ & 115.1 & 1.8 & -0.8325 & $0.7916-0.8750$ & -115.6 & 1.7 \\
\hline 0.9166 & $0.8750-0.9583^{\dagger}$ & 123.2 & 1.6 & -0.9149 & $0.8750-0.9583^{\dagger}$ & -111.9 & 1.9 \\
\hline 0.9994 & $0.9583-1.0416$ & 121.9 & 1.3 & -0.9990 & $0.9583-1.0416$ & -112.2 & 1.8 \\
\hline 1.1127 & $1.0416-1.2083$ & 120.3 & 1.2 & -1.1217 & $1.0416-1.2083$ & -118.6 & 1.8 \\
\hline 1.2876 & $1.2083-1.3750$ & 129.8 & 1.1 & -1.2908 & $1.2083-1.3750$ & -119.8 & 1.7 \\
\hline 1.4572 & $1.3750-1.5417$ & 136.0 & 1.0 & -1.4576 & $1.3750-1.5417$ & -125.7 & 2.2 \\
\hline 1.6221 & $1.5417-1.7083$ & 134.8 & 1.1 & -1.6220 & $1.5417-1.7083$ & -128.5 & 1.8 \\
\hline 1.7900 & $1.7083-1.8750$ & 132.7 & 1.1 & -1.7897 & $1.7083-1.8750$ & -132.9 & 2.7 \\
\hline 1.9741 & $1.8750-2.0833$ & 137.4 & 1.5 & -1.9705 & $1.8750-2.0833$ & -137.4 & 1.5 \\
\hline 2.1838 & $2.0833-2.2917$ & 134.1 & 1.5 & -2.1801 & $2.0833-2.2917$ & -132.4 & 2.0 \\
\hline 2.4630 & $2.2917-2.6667$ & 129.3 & 1.8 & -2.4728 & $2.2917-2.6667$ & -138.1 & 5.0 \\
\hline 3.1024 & $2.6667-3.6667$ & 120.2 & 6.9 & -3.0919 & $2.6667-3.6667$ & -135.5 & 7.0 \\
\hline
\end{tabular}

NOTE. - All values are measured with respect to M82's center. The positions are flux weighted, and the uncertainties were calculated as the rms scatter between the six templates. At M82's distance, $1^{\prime} \approx 1.056 \mathrm{kpc}$.

$\dagger$ Cross-correlation aperture

lar rotation curve. The horizontal bars represent aperture size. All data points without error bars have uncertainties less than $2 \mathrm{~km} \mathrm{~s}^{-1}$ and aperture sizes less than $5^{\prime \prime}$. Table 1 lists the measurements (apertures selected as cross-correlation templates are marked with a dagger).

The observed stellar velocities show the stellar rotation curve is flat on scales of $1-4 \mathrm{kpc}$. This seems to contradict the falling rotation curve suggested by $\mathrm{H} \mathrm{I}$ and $\mathrm{CO}$ emission from the interstellar medium (Sofue et al. 1992; Yun et al. 1993). The gas, however, is severely disrupted by the wind and may also be affected by radial inflow and tides (Young \& Scoville 1984). The stellar data are therefore expected to trace the mass distribution much more reliably.

The rotation curve for M82E appears to decline slightly from $\sim 2 \rightarrow 4 \mathrm{kpc}$. This decline has $\sim 2 \sigma$ significance and may be due to tidal stripping caused by the interaction with M81. The decline in the rotation curve is at the approximate location of a flaring in the stellar disk and the northern $\mathrm{H} \mathrm{I}$ streamer (Yun et al. 1993). No decline is seen in the M82W data. Measurements at larger radii are needed to determine whether or not the falloff is due to tidal stripping.

Telesco et al. (1991) discovered M82's stellar bar with observations at infrared wavelengths and estimated it to be approximately $1 \mathrm{kpc}$ in length. Numerical simulations have shown that galaxy interactions are effective at inducing galactic bars (e.g. Noguchi 1988; Miwa \& Noguchi 1998), and the strong evidence for past tidal interaction observed in M82 makes it an excellent testing ground for theories of bar for- mation. Although it is difficult to observationally determine the origin of M82's bar, the effects of its presence can be seen in our stellar rotation curve as the symmetric, sharp features on either side of the nucleus.

The second bump at $\sim 500 \mathrm{pc}$ may be the result of a super star cluster (seen in the two-dimensional data) that fell on the slit. This cluster, which was identified as cluster ' $z$ ' by McCrady \& Graham (2007), likely dominates the flux from this aperture, and its velocity excess with respect to the main rotation curve likely indicates strong non-circular motion. However, it is puzzling that multiple points define the feature and that no other studies have seen this second peak in the rotation curve. Other possible explanations for the feature are the presence of tightly wound spiral arms and patchy $K$-band extinction.

Our data contain evidence of a "velocity reversal" (velocity decrease) at $\sim 1.0 \mathrm{kpc}$ on both the eastern and western sides of the galaxy. Greve (2011) highlights the existence of a $100 \mathrm{~km} \mathrm{~s}^{-1}$ velocity reversal of gas and/or stars at $\sim 1.0 \mathrm{kpc}$ in $\mathrm{H} \delta$ and $\mathrm{H} \epsilon$ stellar absorption on the eastern side of the galaxy, as well as in ionized gas emission lines at visible and blue wavelengths on the eastern and western sides; interestingly, the feature is apparently absent in near-infrared Ca II stellar absorption. Although the velocity amplitude of our detection is approximately a factor of 10 less, the reversal is present in our data. Local extinction is not likely to be the culprit, as the feature is symmetric with respect to the nucleus. Greve (2011) investigates the hypothesis that the re- 

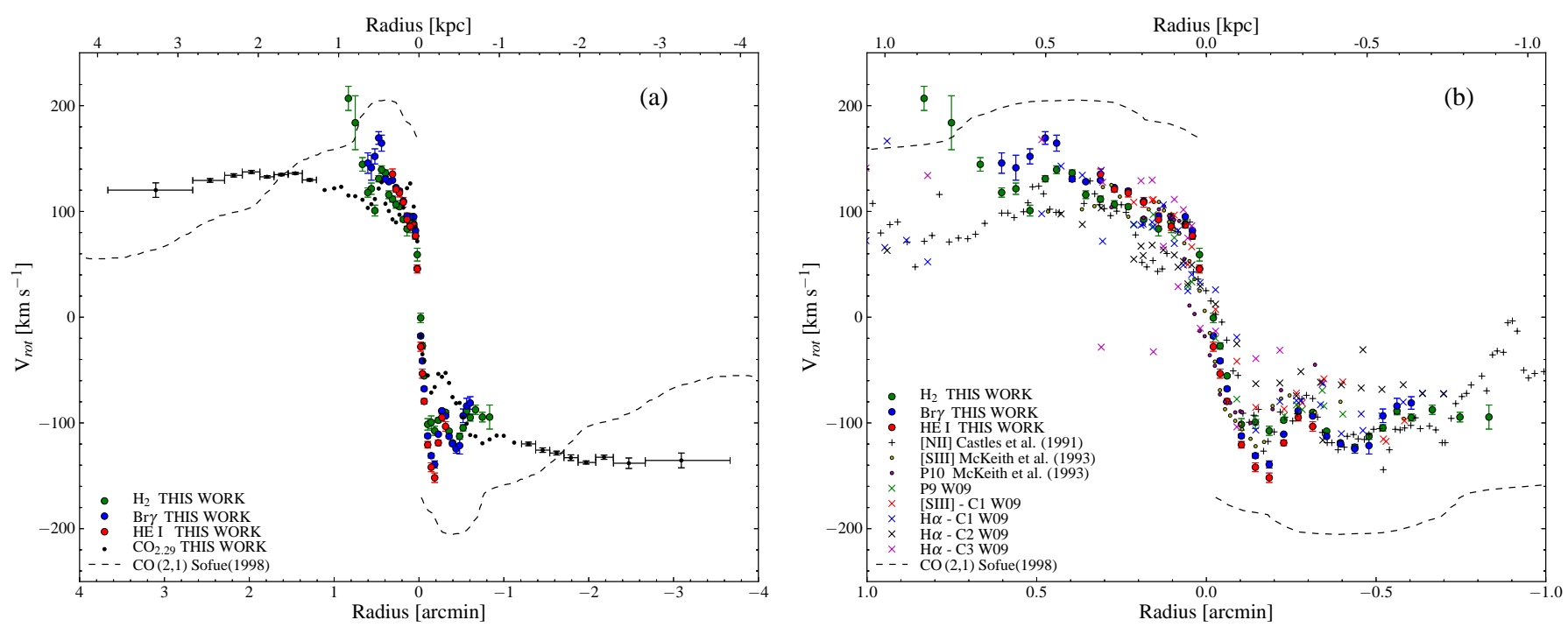

FIG. 4.- (a) The gas and stellar rotation curves from this work and the measurements of CO $(2,1)$ by Sofue (1998). On average, the gas has larger velocities than the stars. (b) Comparison of gas measurements of the inner $1 \mathrm{kpc}$ radius from this work with gas measurements by Castles et al. (1991), McKeith et al. (1993), Westmoquette et al. (W09; 2009), and Sofue (1998). The C labels with the W09 data refer to the different components of an IFU observation.

versal is due to local surface density enhancements associated with spiral arms (Mayya et al.2005), but finds inconsistencies with this picture. For instance, the absence of the velocity reversal in near-infrared Ca II stellar absorption suggests that it is not inherent to the disk, and the blue-wavelength detections of the eastern velocity reversal reach negative velocities, which cannot be explained from the rotation of a stellar disk with embedded corotating spiral arms. Nevertheless, the detection of the reversal in ${ }^{12} \mathrm{CO}$ stellar absorption and the symmetry about the nucleus indicate the feature is present in the stellar population and implies it is due to a local density enhancement, such as spiral arms.

The inset of Figure 3 compares our results for the inner region with Westmoquette et al. (2009) and McKeith et al. (1993). The agreement with Westmoquette et al. (2009) is excellent outside the bar region. There is good agreement with McKeith et al. (1993), as well as some interesting differences. The sharp peaks just off of the nucleus are in agreement on the eastern side but not on the western side. McKeith et al. (1993) measured a larger amplitude for the western peak. Interestingly, the western peak of our gas measurements (described next, and see Figure (4) is in better agreement with McKeith et al. (1993) than our stellar measurements.

\subsection{Gas Emission}

There are several prominent gas emission lines in our data that are characteristic of starburst activity, most notably $\mathrm{Br} \gamma$, $\mathrm{He} \mathrm{I}$, and $\mathrm{H}_{2}$ (Figure 2). The $\mathrm{Br} \gamma$ and $\mathrm{He} \mathrm{I}$ are recombination lines that trace photoionized nebulae, and the $\mathrm{H}_{2}$ is a rovibrational line that traces shocked gas. In contrast to the observed strength of $\mathrm{CO}_{2.29}$ relative to the continuum, which decreases with distance from the nucleus, the emission lines show an increase of intensity with distance to $350 \mathrm{pc}$ radius and a rapid decrease beyond $1 \mathrm{kpc}$ radius. This is in qualitative agreement with the intensity variations measured by Förster Schreiber et al. (2001).

For each aperture listed in Table 1 with sufficient signalto-noise, we used the emsao package within IRAF to measure the velocity offset of each emission line relative to its rest wavelength (Cox 2000). We normalized the emission line rotation curves to the $\mathrm{CO}_{2.29}$ velocity in an aperture $\sim 1.25^{\prime \prime}$ west of the nucleus. Figure 4 compares our gas and stellar rotation curves with the nearly Keplerian gas dynamics measured in CO $(2,1)$ by Sofue (1998). The emission lines used to measure the gas kinematics are only detected out to $1 \mathrm{kpc}$.

On average, our measurements show that the gas moves with higher velocities than the stars inside $1 \mathrm{kpc}$. This can be explained by forces from the stellar bar, which tend to drive the gas towards the center of the galaxy. Figure 4 a compares our gas measurements with our stellar rotation curve and radio observations of CO $(2,1)$ by Sofue (1998). The dramatic decline seen in Sofue (1998) is not seen in the $\mathrm{CO}_{2.29}$ rotation curve. Figure $4 \mathrm{~b}$ compares gas measurements by Castles et al. (1991), McKeith et al. (1993), Westmoquette et al. (W09; 2009), and Sofue (1998) with our measurements. These data cover a wide wavelength range and are in fairly good agreement. There is a large discrepancy in amplitude between Sofue (1998) and the other data. As the depth of an observation is a function of wavelength, these discrepancies may be the result of optical depth variations.

\section{MASS DISTRIBUTION}

The mass of M82 has a much larger contribution from atomic and molecular gas than seen in typical spiral galaxies. Young \& Scoville (1984) estimate that between $30 \%$ and $40 \%$ of the galaxy's mass is in the ISM and that the $\mathrm{H}_{2} / \mathrm{HI}$ mass ratio is approximately 10:1. These authors postulate that the high gas fraction may be due to infalling material that contributes to the available reservoir. The estimated dynamical mass of M82 ranges from several times $10^{9} \rightarrow 10^{10} M_{\odot}$ in the literature (e.g. Burbidge et al.|1964; Crutcher et al. 1978; Young \& Scoville 1984; Goetz et al. 1990; Sofue et al. 1992); however, the substantial dust attenuation and non-circular motions associated with tidal forces and the stellar bar make the estimation of the mass challenging and uncertain.

Figure 5 shows the mass profile based on our stellar kinematic measurements. Similar to the studies mentioned above, we assumed circular motion to calculate the enclosed mass at each point. While non-circular motions are expected in the central region, and in particular in the bar region, circular motion is a good assumption on large scales. At $4 \mathrm{kpc}$ 
the mass distribution appears to be asymptotically approaching $\sim 10^{10} M_{\odot}$. This is likely the total mass of the galaxy, as these measurements are based on stellar motions and extend out to nearly $4 \mathrm{kpc}$ from the nucleus.

Circular motion is not a good assumption in the central region, where the bar and perhaps other asymmetries in the potential produce non-circular motions. Nevertheless, here we estimate the mass within $500 \mathrm{pc}$ is $\lesssim 2 \times$ $10^{9} M_{\odot}$, which is consistent with mass measurements of this region by Young \& Scoville (1984), Goetz et al. (1990), and Greve et al. (2002). This mass estimate may be high by a factor of two based on the relative mass distributions for M82E and M82W shown in Figure 5.

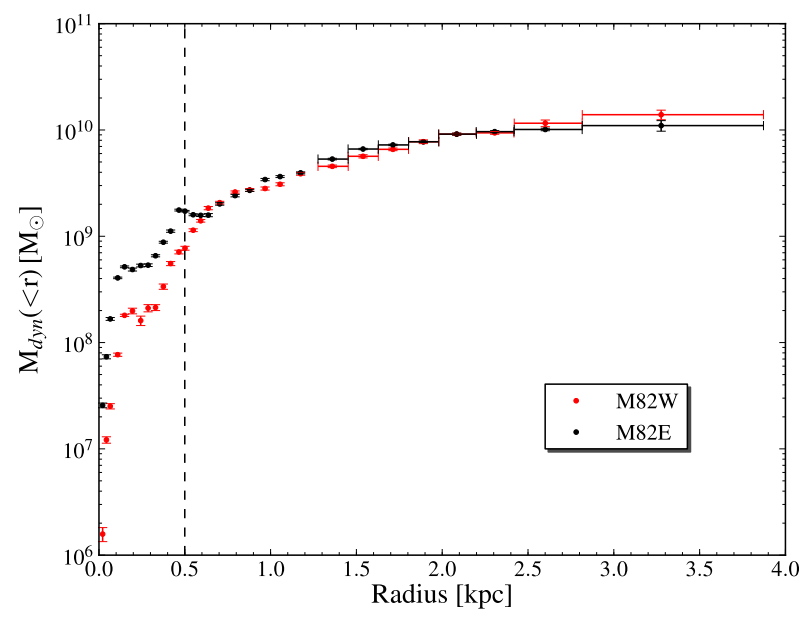

FIG. 5.- Mass profile derived from our stellar rotation curve. Circular motion was assumed in the derivation. The horizontal error bars denote the aperture size of the measurement. The apparent decrease in mass at small radii may be due to the non-circular motion associated with the stellar bar. The superwind is likely launched from the region inside the dashed line, where we estimate the mass is $\lesssim 2 \times 10^{9} M_{\odot}$.

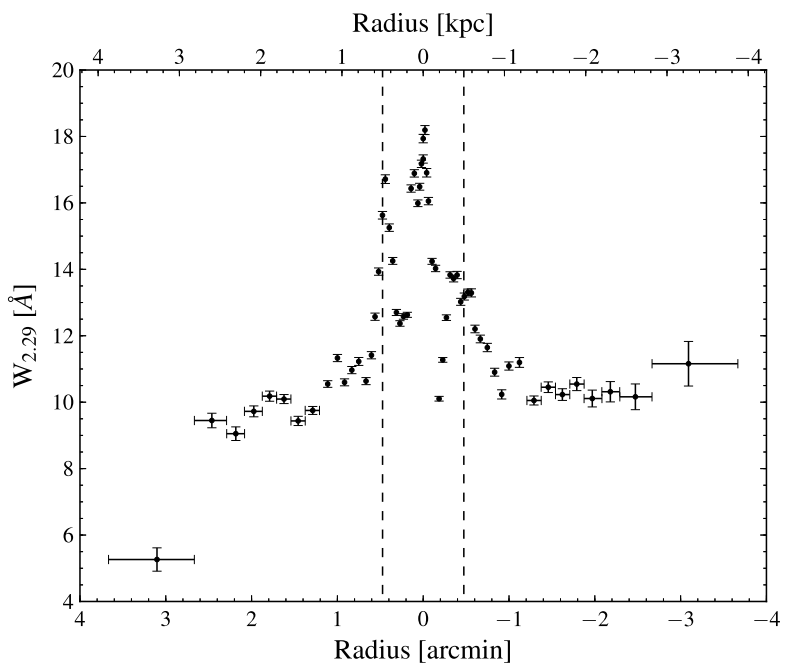

FIG. 6.- $-\mathrm{W}_{2.29}$ as a function of distance from the center of the galaxy. The horizontal error bars indicate the aperture size of the measurement. The peak in $\mathrm{W}_{2.29}$ inside the dashed region is indicative of a stellar population dominated by RSGs.

\section{STELLAR POPULATIONS}

The $\mathrm{CO}$ absorption index in stars increases with increasing stellar luminosity, decreasing effective temperature, and increasing metallicity (Frogel et al. 1978, 1983). Large CO indices are indicative of either a stellar population dominated by RSGs or one dominated by old, metal-rich giants. These two possibilities have led to much debate on the nature of M82's evolved stellar population, particularly in the nucleus (Förster Schreiber et al. 2001). Starburst models with RSG-dominated nuclei, however, provide the best fit to the observed properties of M82 (Rieke et al. 1993; Förster Schreiber et al. 2003).

Our data reveal very deep CO absorption in M82's starburst core. In Figure 6, we show the equivalent width of $\mathrm{CO}_{2.29}$ $\left(\mathrm{W}_{2.29}\right)$ as a function of radius. The data clearly show a significant increase in $\mathrm{W}_{2.29}$ within 500 pc radius. Based on the diagnostic diagrams proposed by Origlia et al. (1993) and Oliva et al. (1995), these results suggest that RSGs dominate the near-infrared continuum throughout the starburst core. At yet smaller radii, Förster Schreiber et al. (2001) found that $\mathrm{W}_{2.29}$ within the central $35 \mathrm{pc}$ is characteristic of RSGs with $T_{\text {eff }}=3600-4200 \mathrm{~K}$.

The dramatic contrast in $\mathrm{W}_{2.29}$ between the starburst core and the stellar disk can be explained by the successive starburst model proposed by Förster Schreiber et al. (2003), in which the disk and the starburst core are composed of two distinct stellar populations.

\section{SUMMARY}

We have used longslit, $K$-band spectroscopy from the LBT to measure the stellar and gas kinematics and study the mass and stellar population distribution in M82. The primary points of this paper can be summarized as follows.

We used ${ }^{12} \mathrm{CO}$ stellar absorption to measure the rotation curve out to nearly $4 \mathrm{kpc}$ on the eastern and western sides of the galaxy. While some previous studies have interpreted $\mathrm{H} \mathrm{I}$ and $\mathrm{CO}$ emission-line position-velocity diagrams as evidence for a declining rotation curve, our data show that it is flat from $1-4 \mathrm{kpc}$ (Figure 3). The rotation curve also contains the signatures of the stellar bar and an apparent "velocity reversal" at $\sim 1.0 \mathrm{kpc}$ on either side of the galaxy that may be the manifestation of symmetric spiral arms.

We measured the rotation curve with the $\operatorname{Br} \gamma$, He I, and $\mathrm{H}_{2}$ emission lines from the interstellar medium out to $1 \mathrm{kpc}$ from the nucleus. These gas rotation curves have velocities $10-50 \mathrm{~km} \mathrm{~s}^{-1}$ greater than the stellar velocities (Figure 4). The gas dynamics are expected to be disrupted more than the stars by the presence of tides and galactic winds, which is the likely origin of the stellar and gas velocity differences.

We used the stellar absorption data to measure the mass distribution (Figure 5) and investigate the stellar populations as a function of radius (Figure 6). We estimate the total dynamical mass of M82 is $\sim 10^{10} M_{\odot}$. The strong variation in $\mathrm{W}_{2.29}$ with radius clearly indicates that the starburst is located within the central 500 pc radius. The superwind is likely launched from this region, and we estimate its mass is $\lesssim 2 \times 10^{9} M_{\odot}$. This mass estimate is somewhat uncertain due to the presence of non-circular motions, but nevertheless provides an upper limit to the gravitational potential of the starburst region.

We thank Mark Westmoquette for comments and supplying data for Figures 3 and 4 . We also appreciate the helpful comments from the referee. We are grateful to Jeff Blackburne and Jill Gerke for taking the data as part of the OSU/RC queue. JPG is grateful for support from an undergraduate research scholarship from the College of Arts and Sciences at the Ohio State University. PM is grateful for support from the NSF via award AST-0705170. TAT is supported in part by NASA 
grant NNXIOADOIG and an Alfred P. Sloan fellowship.

\section{REFERENCES}

Achtermann, J. M., \& Lacy, J. H. 1995, ApJ, 439, 163

Burbidge, E. M., Burbidge, G. R., \& Rubin, V. C. 1964, ApJ, 140, 942

Castles, J., McKeith, C. D., \& Greve, A. 1991, Vistas in Astronomy, 34, 187

Cottrell, G. A. 1977, MNRAS, 178, 577

Cox, A. N. 2000, Allen's Astrophysical Quantities, 4th ed.

Crutcher, R. M., Rogstad, D. H., \& Chu, K. 1978, ApJ, 225, 784

Draine, B. T. 1989, Infrared Spectroscopy in Astronomy, 290, 93

Förster Schreiber, N. M., Genzel, R., Lutz, D., Kunze, D. , \& Sternberg, A. 2001, ApJ, 552, 544

Förster Schreiber, N. M., Genzel, R., Lutz, D., \& Sternberg, A. 2003, ApJ, 599,193

Freedman, W. L., et al. 1994, ApJ, 427, 628

Frogel, J. A., Persson, S. E., Matthews, K., \& Aaronson, M. 1978, ApJ, 220, 75

Frogel, J. A., Persson, S. E., \& Cohen, J. G. 1983, ApJS, 53, 713

Gaffney, N. I., Lester, D. F., \& Doppmann, G. 1995, PASP, 107, 68

Gerke, J. R., Kochanek, C. S., Prieto, J. L., Stanek, K. Z., \& Macri, L. M. 2011, arXiv:1103.0549

Goetz, M., Downes, D., Greve, A., \& McKeith, C. D. 1990, A\&A, 240, 52

Gottesman, S. T., \& Weliachew, L. 1977, ApJ, 211, 47

Greve, A., Wills, K. A., Neininger, N., \& Pedlar, A. 2002, A\&A, 383, 56

Greve, A. 2011, A\&A, 529, A51

Konstantopoulos, I. S., Bastian, N., Smith, L. J., et al. 2009, ApJ, 701, 1015

Lester, D. F., Carr, J. S., Joy, M., \& Gaffney, N. 1990, ApJ, 352, 544

Lo, K. Y., Cheung, K. W., Masson, C. R., et al. 1987, ApJ, 312, 574

Lynds, C. R., i\& Sandage, A. R. 1963, ApJ, 137, 1005

Mayall, N. U. 1960, Annales d'Astrophysique, 23, 344

Mayya, Y. D., Carrasco, L., \& Luna, A. 2005, ApJ, 628, L33

McKeith, C. D., Castles, J., Greve, A., \& Downes, D. 1993, A\&A, 272, 98

McKeith, C. D., Greve, A., Downes, D., \& Prada, F. 1995, A\&A, 293, 703
McCrady, N., \& Graham, J. R. 2007, ApJ, 663, 844

Miwa, T., \& Noguchi, M. 1998, ApJ, 499, 149

Noguchi, M. 1988, A\&A, 203, 259

Oliva, E., Origlia, L., Kotilainen, J. K., \& Moorwood, A. F. M. 1995, A\&A, 301,55

Origlia, L., Moorwood, A. F. M., \& Oliva, E. 1993, A\&A, 280, 536

Puxley, P. J. 1991, MNRAS, 249, 11P

Rieke, G. H., Lebofsky, M. J., Thompson, R. I., Low, F. J., \& Tokunaga, A. T. 1980, ApJ, 283, 24

Rieke, G. H., Loken, K., Rieke, M. J., \& Tamblyn, P. 1993, ApJ, 412, 99

Rubin, V. C., \& Ford, W. K., Jr. 1983, ApJ, 271, 556

Saito, M., Sasaki, M., Kaneko, N., Nishimura, M., \& Toyama, K. 1984, PASJ, 36, 305

Sakai, S., \& Madore, B. F. 1999, ApJ, 526, 599

Sanders, D. B., Mazzarella, J. M., Kim, D.-C., Surace, J. A., \& Soifer, B. T. 2003, AJ, 126, 1607

Sofue, Y. 1998, PASJ, 50, 227

Sofue, Y., Reuter, H.-P., Krause, M., Wielebinski, R., \& Nakai, N. 1992, ApJ, 395, 126

Sofue, Y., \& Rubin, V. 2001, ARA\&A, 39, 137

Telesco, C. M., Campins, H., Joy, M., Dietz, K., \& Decher, R. 1991, ApJ, 369,135

Vacca, W. D., Cushing, M. C., \& Rayner, J. T. 2003, PASP, 115, 389

Westmoquette, M. S., Smith, L. J., Gallagher, J. S., III, et al. 2009, ApJ, 696, 192

Young, J. S., \& Scoville, N. Z. 1984, ApJ, 287, 153

Yun, M. S., Ho, P. T. P., \& Lo, K. Y. 1993, ApJ, 411, L17

Yun, M. S., Ho, P. T. P., \& Lo, K. Y. 1994, Nature, 372, 530 